\title{
Learning Curve and Complications Experience of Oblique Lateral Interbody Fusion : A Single-Center 143 Consecu- tive Cases
}

\author{
Bu Kwang Oh, ${ }^{1}$ Dong Wuk Son, ${ }^{1,2}$ Su Hun Lee, Jun Seok Lee, ${ }^{1}$ Soon Ki Sung, ${ }^{1,2}$ Sang Weon Lee, ${ }^{1,2}$ Geun Sung Song ${ }^{1,2}$ \\ Department of Neurosurgery,' Research Institute for Convergence of Biomedical Science and Technology, Pusan National University Yangsan \\ Hospital, Yangsan, Korea \\ Deparment of Neurosurgery, ${ }^{2}$ School of Medicine, Pusan National University, Yangsan, Korea
}

Objective : Oblique lateral interbody fusion (OLIF) is becoming the preferred treatment for degenerative lumbar diseases. As beginners, we performed 143 surgeries over 19 months. In these consecutive cases, we analyzed the learning curve and reviewed the complications in our experience.

Methods : This was a retrospective study; however, complications that were well known in the previous literature were strictly recorded prospectively. We followed up the changes in estimated blood loss (EBL), operation time, and transient psoas paresis according to case accumulation to analyze the learning curve.

Results : Complication-free patients accounted for $43.6 \%$ (12.9\%, early stage 70 patients and $74.3 \%$, late stage 70 patients). The most common complication was transient psoas paresis $(n=52)$. Most of these complications occurred in the early stages of learning. C-reactive protein normalization was delayed in seven patients (4.89\%). The operation time showed a decreasing trend with the cases; however, EBL did not show any significant change. Notable operation-induced complications were cage malposition, vertebral body fracture, injury to the ureter, and injury to the lumbar vein.

Conclusion : According to the learning curve, the operation time and psoas paresis decreased. It is important to select an appropriately sized cage along with clear dissection of the anterior border of the psoas muscle to prevent OLIF-specific complications.

Key Words : Intervertebral disc · Retroperitoneal space · Spinal fusion/adverse effect · Psoas muscle · Learning curve.

\section{INTRODUCTION}

Since first described by Silvestre et al. ${ }^{25}$, oblique lateral interbody fusion (OLIF) has been performed in degenerative lumbar diseases. Since OLIF uses a potential retroperitoneal space, we can access the intervertebral disc relatively easily with little bleeding and muscle injury ${ }^{4}$. In addition, this method accesses the paravertebral space and does not require access to the prevertebral space, which may accompany great vessel injury or damage to the prevertebral plexus ${ }^{23)}$. Addition-

- Received : December 10, 2020 • Accepted : January 13, 2021

- Address for reprints : Dong Wuk Son

Department of Neurosurgery, Pusan National University Yangsan Hospital, 20 Geumo-ro, Mulgeum-eup, Yangsan 50612, Korea

Tel : +82-55-360-2126, Fax : +82-55-360-2156, E-mail : md6576@naver.com, ORCID : https://orcid.org/0000-0002-9154-1923

This is an Open Access article distributed under the terms of the Creative Commons Attribution Non-Commercial License (http://creativecommons.org/licenses/by-nc/4.0) which permits unrestricted non-commercial use, distribution, and reproduction in any medium, provided the original work is properly cited. 
ally, this method uses the prepsoas approach and psoas retraction rather than the transpsoas method, and there is less chance of potential injury to the lumbar plexus ${ }^{19)}$. These advantages help beginners gain easier access, thereby lowering the risk of intraoperative musculoligamentous injury or bleeding. We can predict that the learning curve for OLIF might be short; however, information related to this is lacking ${ }^{3,20)}$. Hence, our consecutive cases could show the learning curve for complications as well as changes in operation time and estimated blood loss (EBL). We have also described how serious OLIF-specific complications could be managed.

\section{MATERIALS AND METHODS}

\section{Patient demographics}

The study protocol was approved by the Institutional Review Board of Pusan National University Yangsan Hospital (IRB No. 05-2020-256), which waived the requirement for informed consent due to the retrospective nature of this study. We retrospectively evaluated 143 consecutive patients who underwent OLIF between April 2018 and October 2019. The inclusion criteria were as follows : 1) severe lumbar spinal stenosis (grade 3-4), lumbar spondylolisthesis, adjacent segment disease, and other lumbar degenerative diseases with segmental instability and 2) ineffective conservative treatment for 6 months. All patient comorbidities, American Society of Anesthesiologists Classification, bone mineral density, and body mass index were examined before surgery.

\section{Operative technique}

Three authors performed the operation. The patient was placed in the right lateral decubitus position, and under fluoroscopic control, the level of the disc was marked on the skin in the true lateral view. An oblique skin incision was made 3-5 $\mathrm{cm}$ ventral to the anterior margin of the vertebral body. After dissecting the abdominal muscles, the peritoneal fat layer was confirmed, and the peritoneum was retracted anteriorly. After touching the target disc, the level was revalidated using a fluoroscope. At the anterior border of the psoas muscle, the plane between the annulus fibrosus and psoas muscle was dissected, taking care not to damage the muscle bundle. Following this, the muscle bundle was retracted posteriorly, and a retractor was applied. A self-fixing pin was inserted after dissecting the muscle attached to the upper vertebral body to secure the view of the pin insertion site, to minimize potential segmental artery damage. After sufficient annulotomy, a discectomy was performed using a shaver. After discectomy, the cage height was determined using trials of various sizes. After inserting a cage packed with demineralized bone matrix into the disc space, the abdominal wall was sutured layer by layer and closed. After the anterolateral procedure, posterior lumbar stabilization was performed with percutaneous pedicle screw (PPS) fixation or open pedicle screw fixation. In most cases, indirect decompression was performed, and decompressive laminectomy was performed as per the following indications : 1) prominent disc protrusion or sequestration with obvious segmental instability and 2) severe spinal stenosis (grade IV) $)^{26)}$.

\section{Assessment of complications}

In each case, the complications that were well known in the previous literature were strictly recorded prospectively and analyzed retrospectively ${ }^{3,9}$. Complications were classified as approach-related complications or medical complications (Table 1).

\section{Prolonged C-reactive protein (CRP) normalization}

Our institution determined the patient's discharge from the hospital when CRP was normalized (CRP $<0.5 \mathrm{mg} / \mathrm{dL}$ ). If CRP did not normalize, the hospital stay was extended for follow-up. If CRP rebounded without other infectious causes (e.g., urinary tract infection, pneumonia, etc.), additional antibiotics were administered until CRP normalization ${ }^{13,16)}$.

\section{Operation time, EBL, and transient psoas paresis according to the learning curve}

We included only the most performed operation types (1or 2-level OLIF with PPS) and excluded the 3-level operation and open screw cases to analyze the learning curve. We classified the patients into four groups according to level and decompressive laminectomy : 1-level with decompression $(1 \mathrm{Lv}+\mathrm{D})$ or indirect decompression (1Lv-D) and 2-levels with decompression $(2 \mathrm{Lv}+\mathrm{D})$ or indirect decompression (2Lv-D). We followed up the changes in transient psoas paresis, operation time, and EBL in consecutive surgeries.

\section{Statistical analysis}

For each of the four groups, the changes in EBL and opera- 
tion time, according to consecutive cases, were analyzed using Spearman's rank correlation coefficient. For 1-level or 2-level OLIF, the change in psoas paresis according to consecutive cases was analyzed using binomial logistic regression analysis.

Table 1. Operation induced complications

\begin{tabular}{|c|c|}
\hline & Value \\
\hline \multicolumn{2}{|l|}{ Approach related complications $(n=157)$} \\
\hline \multicolumn{2}{|l|}{ Anatomical structural complications } \\
\hline Peritoneal laceration & 2 \\
\hline Vascular injury & 2 \\
\hline Ureteral injury & 1 \\
\hline \multicolumn{2}{|l|}{ Neurologic complications } \\
\hline Retrograde ejaculations & 0 \\
\hline Genitofemoral nerve injury & 5 \\
\hline Postoperative remnant symptom & 30 \\
\hline New-onset nerve root symptom & 4 \\
\hline Peroneal nerve palsy & 1 \\
\hline Transient psoas paresis & 52 \\
\hline Grade 4 & 39 \\
\hline Grade 3 & 13 \\
\hline \multicolumn{2}{|l|}{ Infectious complications } \\
\hline Superficial wound problem & 3 \\
\hline Organ space infection & 1 \\
\hline Delayed CRP normalization & 7 \\
\hline \multicolumn{2}{|l|}{ Instrumental complications } \\
\hline Cage subsidence & 8 \\
\hline Cage malposition & 2 \\
\hline Hardware failure & 0 \\
\hline Vertebral body fracture & 2 \\
\hline \multicolumn{2}{|l|}{ Approach site complication } \\
\hline Incision site pain & 34 \\
\hline Abdominal protrusion & 3 \\
\hline \multicolumn{2}{|l|}{ Medical complication $(n=11)$} \\
\hline Atelectasis & 0 \\
\hline Pneumonia & 0 \\
\hline PTE & 0 \\
\hline DVT & 0 \\
\hline Delirium & 1 \\
\hline UTI & 1 \\
\hline Ileus & 9 \\
\hline
\end{tabular}

CRP : C-reactive protein, PTE : pulmonary thromboembolism, DVT : deep vein thrombosis, UTI : urinary tract infection
Analyses were performed using IBM SPSS Statistics version 26.0 (IBM Co., Armonk, NY, USA) and MedCalc version 19.6 (MedCalc Software Ltd., Ostend, Belgium).

\section{RESULTS}

Of the 214 segments that were operated, 1-level OLIF was performed for seventy-seven patients, 2-level OLIF was performed for sixty-one patients, and 3-level OLIF was performed for five patients. Details of the patient characteristics and operation data are shown in Tables 2-4.

A total of 168 complications occurred during the 143 OLIF procedures at our hospital. There were 11 cases of medical complications (Table 1), none of which involved serious medical complications. There were 157 cases of approach-related complications (Table 1). Half of them (52\%) had mild neurological complications, such as transient psoas paresis (52 pa-

Table 2. Demographic characteristics

\begin{tabular}{lc}
\hline & Value \\
\hline Number of patients & 143 \\
Sex, male : female & $67: 78$ \\
\hline Age (years) & $66.25 \pm 7.02$ \\
BMD & $-0.27 \pm 1.62$ \\
BMl (kg/m ${ }^{2}$ ) & $25.70 \pm 3.40$ \\
Diagnosis & \\
Spondylolisthesis & 129 \\
Spinal stenosis & 81 \\
HNP & 96 \\
Revision surgery & 34 \\
ASA class & \\
Class 1 & 22 \\
Class 2 & 119 \\
Class 3 & 2 \\
Co-morbidities & \\
Hypertension & 81 \\
Diabetes mellitus & 40 \\
Osteoporosis & 9 \\
Cardiovascular disease & 4 \\
History of cancer & 2 \\
\hline
\end{tabular}

Values are presented as mean \pm standard deviation or number (\%). BMD : bone mineral density, BMI : body mass index, HNP : herniated nucleus pulposus, ASA : American Society of Anesthesiologists Classification 
Table 3. Operation data (level)

\begin{tabular}{|c|c|}
\hline & Value \\
\hline \multicolumn{2}{|l|}{1 level } \\
\hline L1-2 & 1 \\
\hline L2-3 & 2 \\
\hline L3-4 & 9 \\
\hline$\lfloor 4-5$ & 61 \\
\hline L5-S1 & 4 \\
\hline Total & 77 \\
\hline \multicolumn{2}{|l|}{2 level } \\
\hline L1-3 & 1 \\
\hline L2-4 & 1 \\
\hline L3-5 & 46 \\
\hline L2-3, L4-5 & 3 \\
\hline L4-S1 & 10 \\
\hline Total & 61 \\
\hline \multicolumn{2}{|l|}{3 level } \\
\hline L1-4 & 0 \\
\hline L2-5 & 5 \\
\hline L3-S1 & 0 \\
\hline Total & 5 \\
\hline \multicolumn{2}{|l|}{ Total } \\
\hline L1-2 & 2 \\
\hline L2-3 & 12 \\
\hline L3-4 & 61 \\
\hline$\lfloor 4-5$ & 125 \\
\hline L5-S1 & 14 \\
\hline Total & 214 \\
\hline
\end{tabular}

Table 4. Operation data (screw \& decompression)

\begin{tabular}{lc}
\hline & Value \\
\hline Posterior fixation & 133 \\
Percutaneous pedicle screw & 10 \\
Open screw & \\
Posterior decompression & \\
Performed & 37 \\
1-level & 39 \\
2-level & 3 \\
3-level & 64 \\
Non-performed & \\
\hline
\end{tabular}

tients) and postoperative remnant pain (30 patients). Complication-free patients accounted for $43.6 \%$ of the patients; the proportion of complication-free patients was $12.9 \%$ in the early stage (70 patients), whereas that in the late stage (70 patients) was 74.3\% (Fig. 1).

Peritoneum laceration occurred in early cases (case \#7 and case \#14). Both patients had a history of abdominal surgery. In both cases, a general surgeon evaluated the bowel injury and sutured the lacerated peritoneum. Both patients recovered without postoperative complications such as bowel injury or peritonitis.

The mean CRP normalization period for 146 patients was 16 days. A total of seven patients (4.8\%) had a prolonged CRP normalization period (Table 5). One patient (case \#64) was discharged with normal CRP $(0.5 \mathrm{mg} / \mathrm{dL})$ but elevated erythrocyte sedimentation rate (ESR) $(60 \mathrm{~mm} / \mathrm{h}$; normal range $0-15 \mathrm{~mm} / \mathrm{h}$ ) on postoperative day 16 . At 5 months postoperatively, the patient was re-admitted to evaluate high ESR, CRP $1.34 \mathrm{mg} / \mathrm{dL}$, and persistent back pain. Chronic osteomyelitis was confirmed by contrast-enhanced magnetic resonance imaging (MRI) and bone scan. There was no prominent abscess around the paravertebral space and epidural space, so revision for abscess drainage was not performed. The patient was discharged after 10 weeks of antibiotic treatment with normal ESR, CRP, and resolution of the infection confirmed on an MRI.

Two cases required revision surgery due to cage malposition (case \#62) and vertebral body fracture (case \#82). Two cases required interventions by a urologist and a vascular surgeon (injury to the ureter [case \#97] and injury to the lumbar vein [case \#63]). Each case is described in further detail in the OLIF-specific complication cases section.

\section{Learning curve}

Transient psoas paresis, which occurred in 52 patients, was the most common complication. Grade 3 and grade 4 psoas muscle weakness occurred in 13 and 39 patients, respectively. In the binary logistic regression analysis, the occurrence of psoas paresis was significantly decreased according to case accumulation in the two groups $(1 \mathrm{Lv}, 2 \mathrm{Lv})$ (Fig. 2). All patients recovered to grade 5 . A total of 51 patients recovered within a week and the others recovered within 2 weeks.

In the indirect decompression cases, there was a trend of decreasing operation time according to case accumulation (1Lv-D, $p=0.057$; 2Lv-D, $p=0.071)$. However, when decompres- 


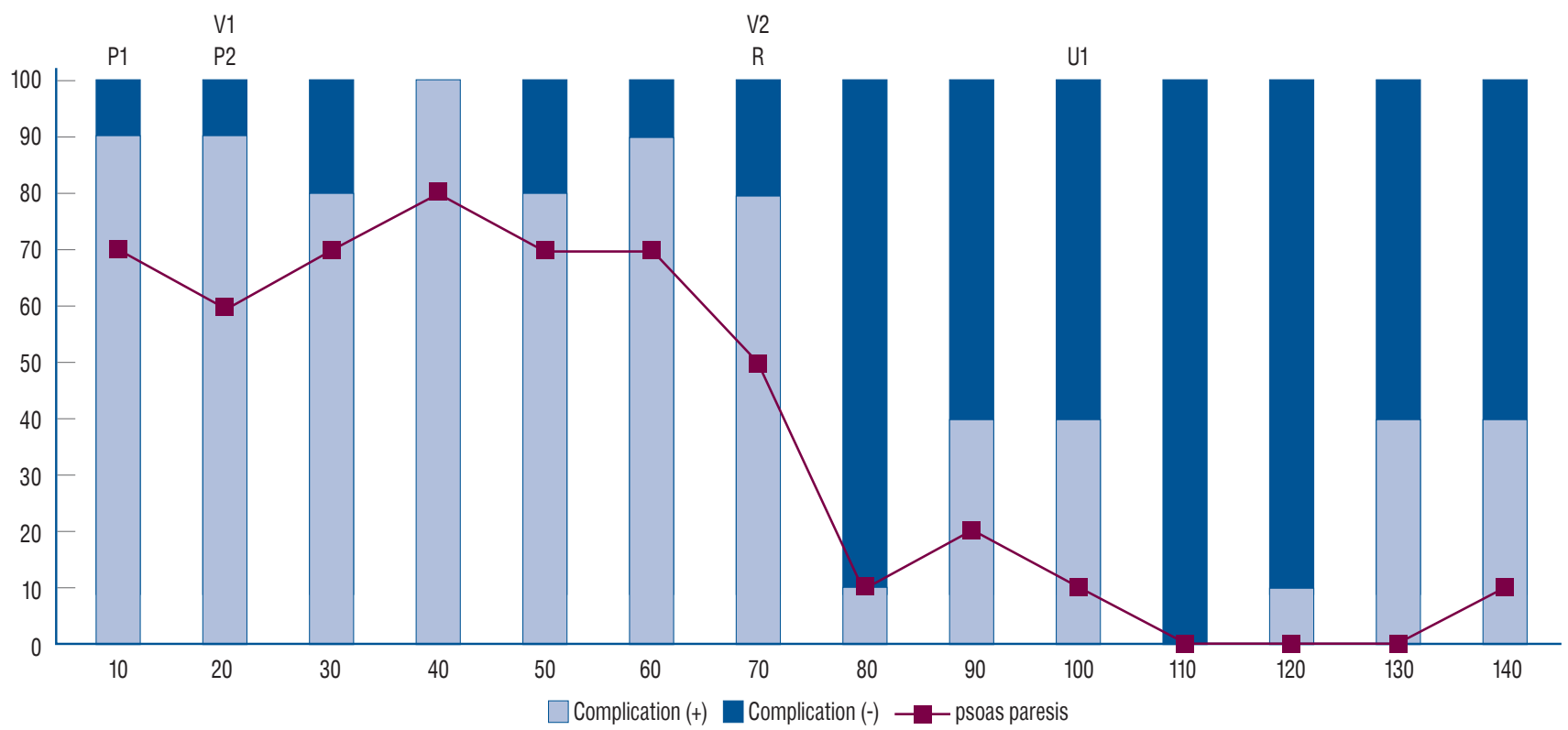

Fig. 1. Changes in complications with case accumulation. The proportion of complications was relatively high in the initial 70 patients, and psoas paresis was the most common complication. The incidence of complications significantly decreased in the 70 patients in the late stage. P1 and P2: two cases of peritoneal laceration, V1 and V2 : two cases of vascular injury, R1 and R2 : two cases of reoperation (R1 : cage repositioning, R2 : vertebral body fracture), $\mathrm{U} 1$ : one case of injury to the ureter.

Table 5. Prolonged CRP normalization and organ space infection

\begin{tabular}{|c|c|c|c|c|c|c|c|c|c|c|c|}
\hline Case & Sex & Age & DM & BMI & ASA & Level & $\begin{array}{c}\text { Post. } \\
\text { Decomp }\end{array}$ & $\begin{array}{c}\text { Post. } \\
\text { Extension }\end{array}$ & $\begin{array}{c}\text { EBL/ } \\
\text { transfusion }\end{array}$ & $\begin{array}{l}\text { Previous } \\
\text { op }\end{array}$ & $\begin{array}{c}\text { Hospital } \\
\text { day }\end{array}$ \\
\hline \multicolumn{12}{|c|}{ Prolonged CRP normalization } \\
\hline$\# 15$ & M & 66 & - & 23.4 & 2 & 2 & & & 200/- & & 20 \\
\hline$\# 21$ & $\mathrm{~F}$ & 74 & + & 28.1 & 2 & 1 & + & & $400-1$ & & 24 \\
\hline \#26 & $\mathrm{F}$ & 69 & + & 25.2 & 2 & 2 & + & & $500 / 2$ pack & & 25 \\
\hline$\# 27$ & $\mathrm{~F}$ & 60 & + & 34.6 & 2 & 1 & & +1 & $300 /-$ & L34 fusion & 47 \\
\hline \#31 & M & 60 & + & 25.5 & 2 & 2 & & +2 & $500 /-$ & L234 fusion & 24 \\
\hline \#36 & $F$ & 71 & + & 29.3 & 2 & 2 & + & & $400 /-$ & Discectomy & 22 \\
\hline \#48 & M & 70 & - & 25.2 & 2 & 3 & + & +1 & $800 / 2$ pack & & 34 \\
\hline \multicolumn{12}{|c|}{ Organ space infection } \\
\hline \#64 & M & 73 & + & 25.9 & 2 & 2 & + & & $500 /-$ & & $16+10$ weeks \\
\hline
\end{tabular}

CRP : C-reactive protein, DM : diabetes mellitus, BMI : body mass index, ASA : American Society of Anesthesiologists Classification, Post. Decomp : posterior decompressive laminectomy, Post. Extension : level of posterior fixation in addition to the oblique lateral interbody fusion level, EBL : estimated blood loss, previous op : previous operation, $\mathrm{M}$ : male, $\mathrm{F}$ : female

sion laminectomy was performed concurrently, the change in operation time according to the accumulation of cases was not significant $(1 \mathrm{Lv}+\mathrm{D}, p=0.55 ; 2 \mathrm{Lv}+\mathrm{D}, p=0.387$; Fig. 3 ). EBL showed no difference according to case accumulation in all groups (Fig. 3).

\section{OLIF-specific complication cases}

\section{Cage malposition}

There were two cases of new contralateral root symptoms, with the cage located near the right neural foramen. In case \#54, new contralateral root symptoms occurred at the level of L4/5. It was challenging to create sufficient orthogonal angu- 

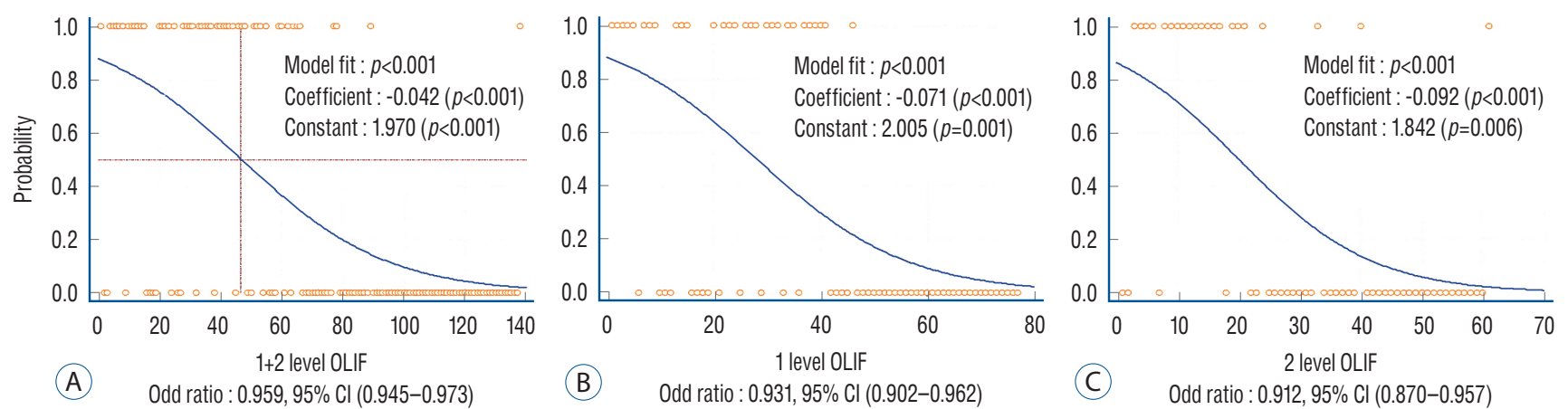

Fig. 2. Binomial logistic regression curve for the change in psoas paresis with case accumulation. A : 1+2-level OLIF. B : 1-level OLIF. C : 2-level OLIF. In both 1- and 2-level OLIF, psoas paresis decreased significantly according to the accumulation of cases. OLIF : oblique lateral interbody fusion, $\mathrm{Cl}$ : confidence interval.

lation due to the high iliac crest. The inserted OLIF cage with an insufficient orthogonal angulation was located near the right neural foramen, and the remnant disc was bulging into the right neural foramen, resulting in a contralateral root symptom. As the disc was absorbed spontaneously, symptoms completely improved four months postoperatively. In case \#62, the patient required revision surgery. The patient had a lesion on the L5/lumbarization of S1. Due to the distal bifurcation of the iliac artery and vein, the OLIF 25 approach (lateral to common iliac vein approach) was performed instead of the OLIF 51 approach (between the bifurcation approach). In this case, it was also challenging to create sufficient orthogonality due to the iliac crest. In the intraoperative $\mathrm{C}$-arm image at the primary surgery, the right posterior radiopaque indicator of the cage was located inside the lower vertebral body posterior margin and inter-pedicle line. However, postoperative computed tomography (CT) performed to evaluate the contralateral root symptom after the primary surgery. We found that the cage was located on the right neural foramen. Due to the orientation of the footprint of the OLIF cage, we preferred a posterior approach rather than an abdominal approach for repositioning the cage. A paramedian approach was performed by connecting the existing percutaneous screw incision for the right PPS. After the rod was removed, the pars interarticularis was partially removed to secure the L5 root. The root was severely compressed by the malpositioned cage. After retraction of the root to the cephalic position, the cage was repositioned using the impactor while monitoring with the C-arm. Following this, the rod was fixed with compression. After the operation, the pain in the right lower extremity partially improved; however, residual numbness persisted (Fig. 4).

\section{Vertebral body fracture}

Coronal vertebral body fractures of the L3 vertebral body occurred in 2-level OLIF (L3/4/5) in female patients with osteoporosis. One patient underwent conservative treatment because segmental stability was maintained (case \#36); however, in one patient, segmental kyphosis progressed, and revision was performed (case \#82). For revision, screw fixation was added to L2, and posterolateral fusion was performed. Following this, the patient's pain decreased, and no additional kyphotic changes occurred (Fig. 5).

\section{Injury to the ureter}

The patient previously underwent L3-5 fusion surgery (case \#97). The patient complained of severe back and leg pain due to L3-4 non-union and L3 bilateral screw loosening. OLIF was performed for L3-4 fusion. There was no problem until the discectomy after the retroperitoneal approach. However, we found that the muscle bundle-like tissue was crushed while pulling out the trail. We asked a urologist to evaluate the possibility of damage to the ureter. Indocyanine green was injected intravenously to confirm any injury to the ureter. Purple urine leaked at the site of injury. A double J catheter was inserted into the ureter at the site of injury, and primary closure was performed by a urologist. The patient was sufficiently hydrated after surgery. However, acute pyelonephritis occurred twice, involving the left kidney. The double J catheter was changed two months postoperatively, and it was removed four months postoperatively. After removal of the catheter, there was mild stenosis at the left proximal ureter anastomosis in retrograde pyelography; however, the dye passed completely without resistance (Fig. 6). 
Single-Center OLIF Experiences | Oh BK, et al.
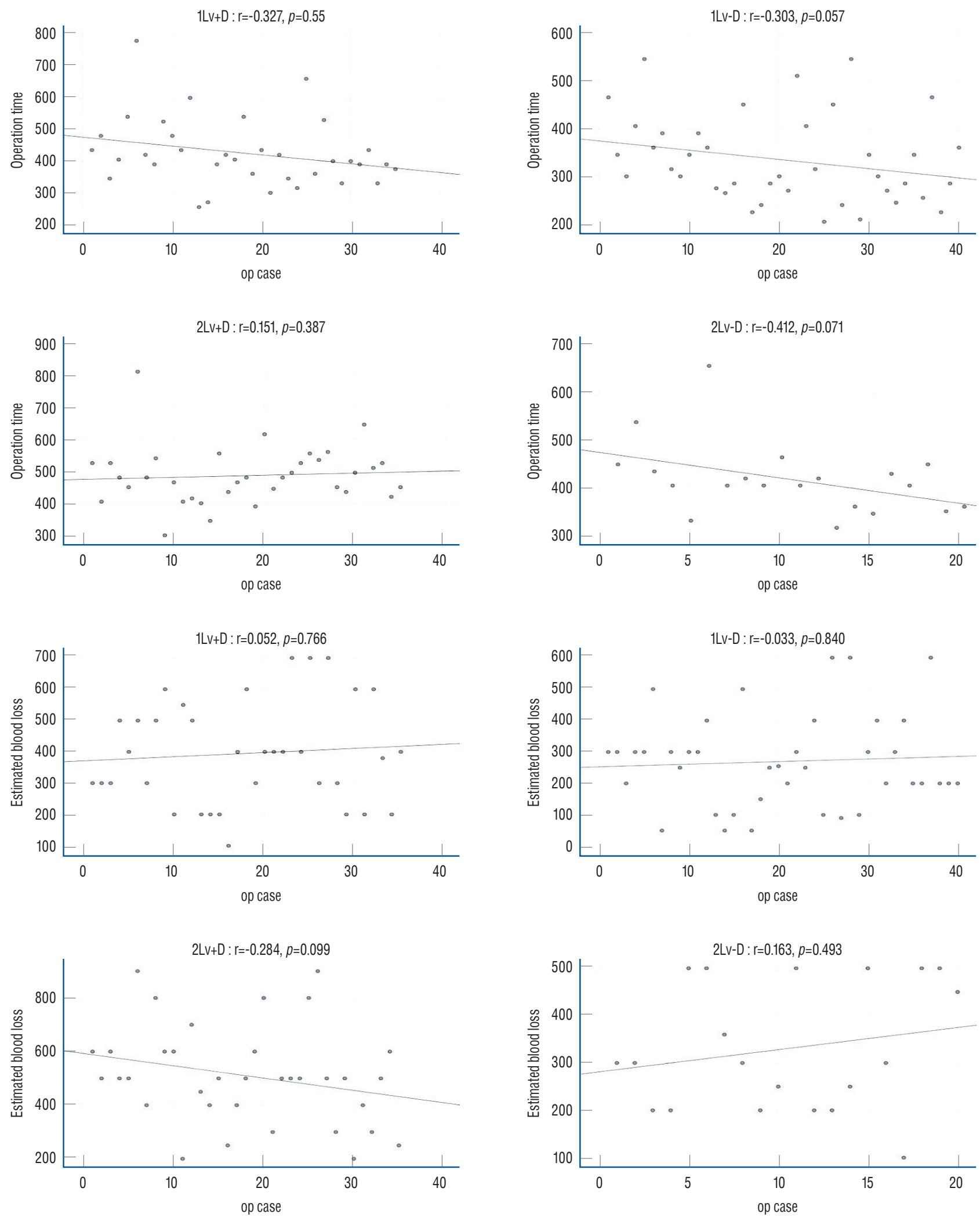

Fig. 3. Scatter plot of the change in operation time (upper 4 graphs) and estimated blood loss (lower 4 graphs) according to consecutive cases in four groups. R : Spearman's rank correlation coefficient, 1Lv : 1-level oblique lateral interbody fusion (OLIF), 2Lv : 2-level OLIF, +D : with decompressive laminectomy, $-\mathrm{D}$ : indirect decompression. 

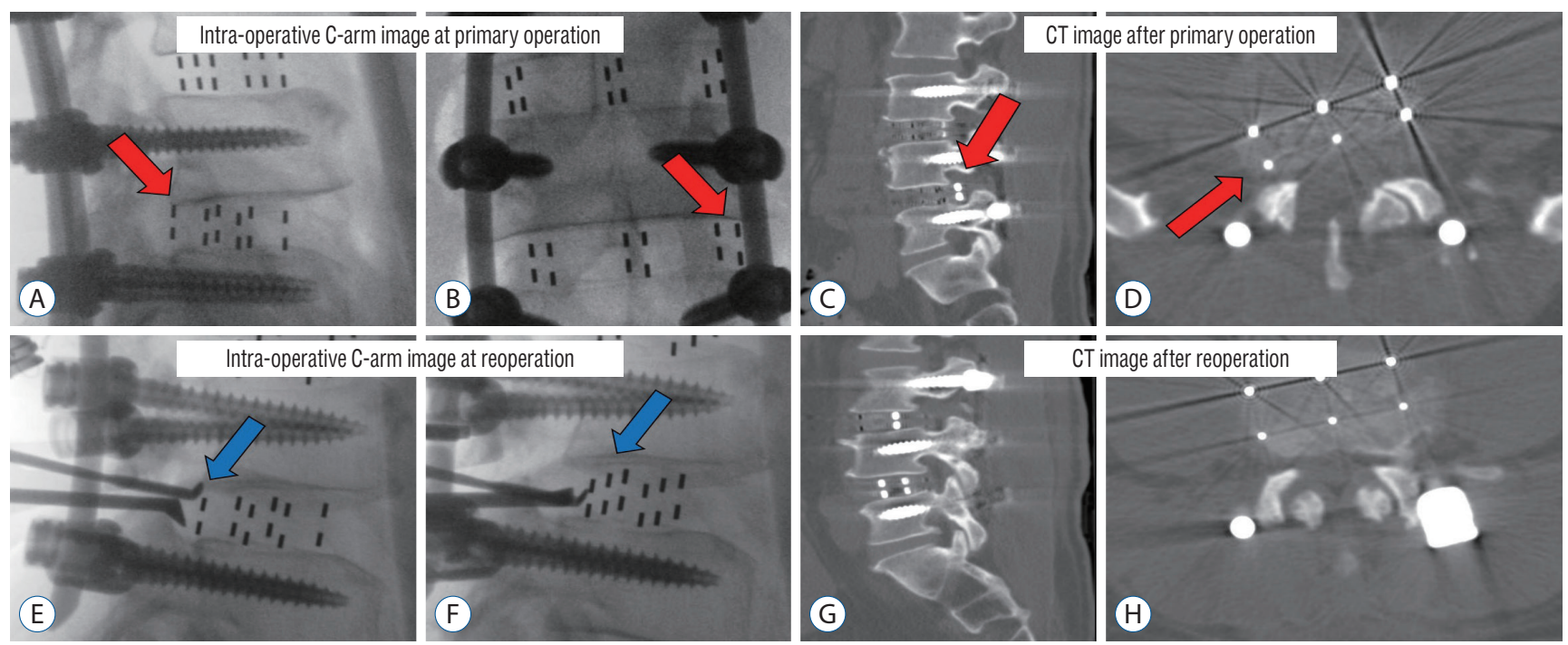

Fig. 4. A-D : Case of cage malposition (red arrow) requiring revision surgery. Intraoperative C-arm image at the primary operation. A : The posterior margin of the cage is located inside the posterior margin of the vertebral body. B: The right lateral margin of the cage is located on the interpedicle line. CT image after the primary operation. C and D : Cage is located in the right neural foramen. $E$ and $F$ : Intraoperative cage reposition (blue arrow) using $\mathrm{C}$-arm image at reoperation. After cephalic retraction of the root $(\mathrm{E})$, the impactor is placed on the cage and repositioned under the $\mathrm{C}$-arm guide $(\mathrm{F})$. $\mathrm{G}$ and $\mathrm{H}$ : CT image after reoperation. Cage repositioning confirmed. $\mathrm{CT}$ : computed tomography.
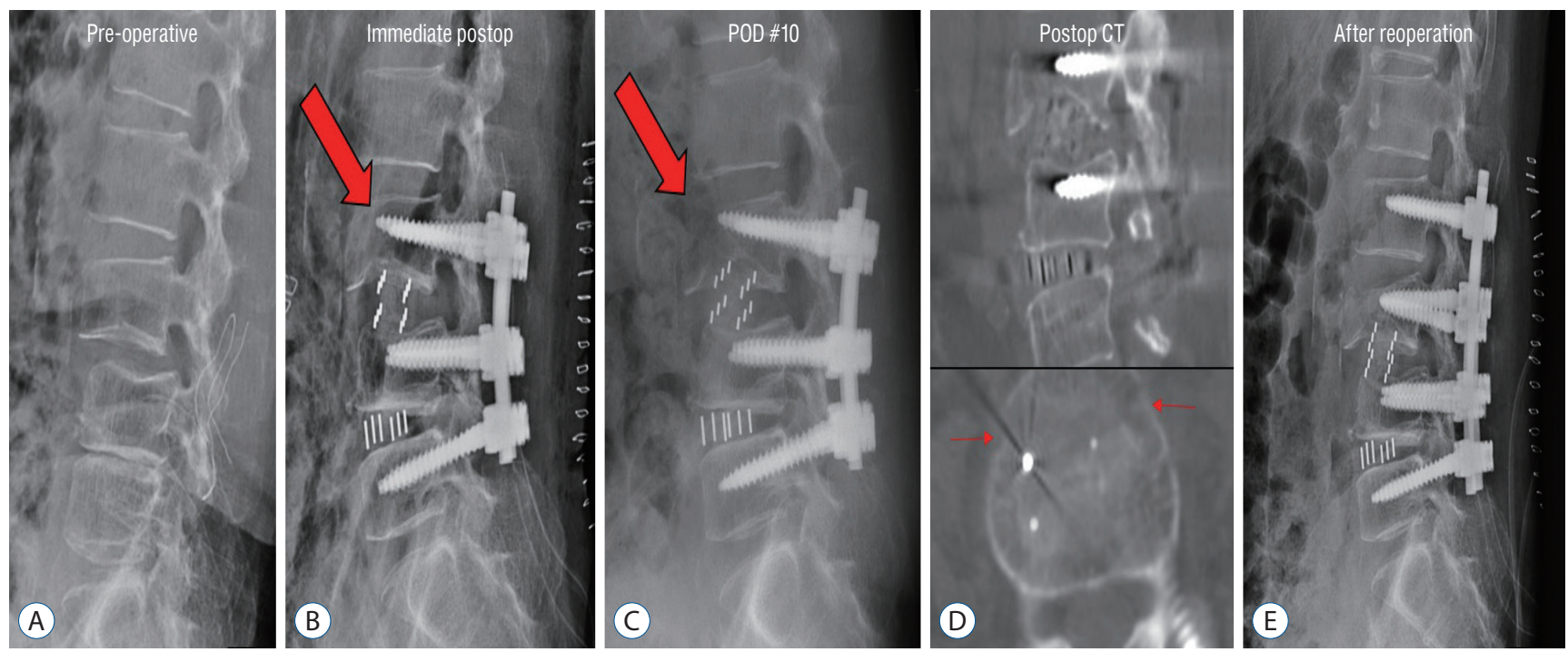

Fig. 5. Case of vertebral body fracture (red arrow) requiring revision surgery. Preoperative (A), Immediate postoperative (B); L3 anterior vertebral body fracture. C : POD 10 day (POD \#10); segmental kyphosis progressed. D : Postoperative CT; the coronal vertebral body fracture of $L 3$. E : After reoperation; posterior fixation extending to $L 2$. POD : postoperative day, $C T$ : computed tomography.

\section{Injury to the lumbar vein}

Injury to the lumbar vein occurred in a female patient who underwent a hysterectomy 10 years ago. There was some adhesion in the retroperitoneal approach due to the previous hysterectomy, and the fat-peritoneal layer did not naturally fall into the anterior medial area, thereby requiring manual retraction. The second assistant retracted the fat-peritoneal layer without visual access, and the right-angle retractor reached the anterior border of the vertebral body, resulting in avulsion of the lumbar vein. We sought help from a general vascular surgeon. After additional dissection of the anterior vertebral border to obtain the field of view, a hemostatic clip 

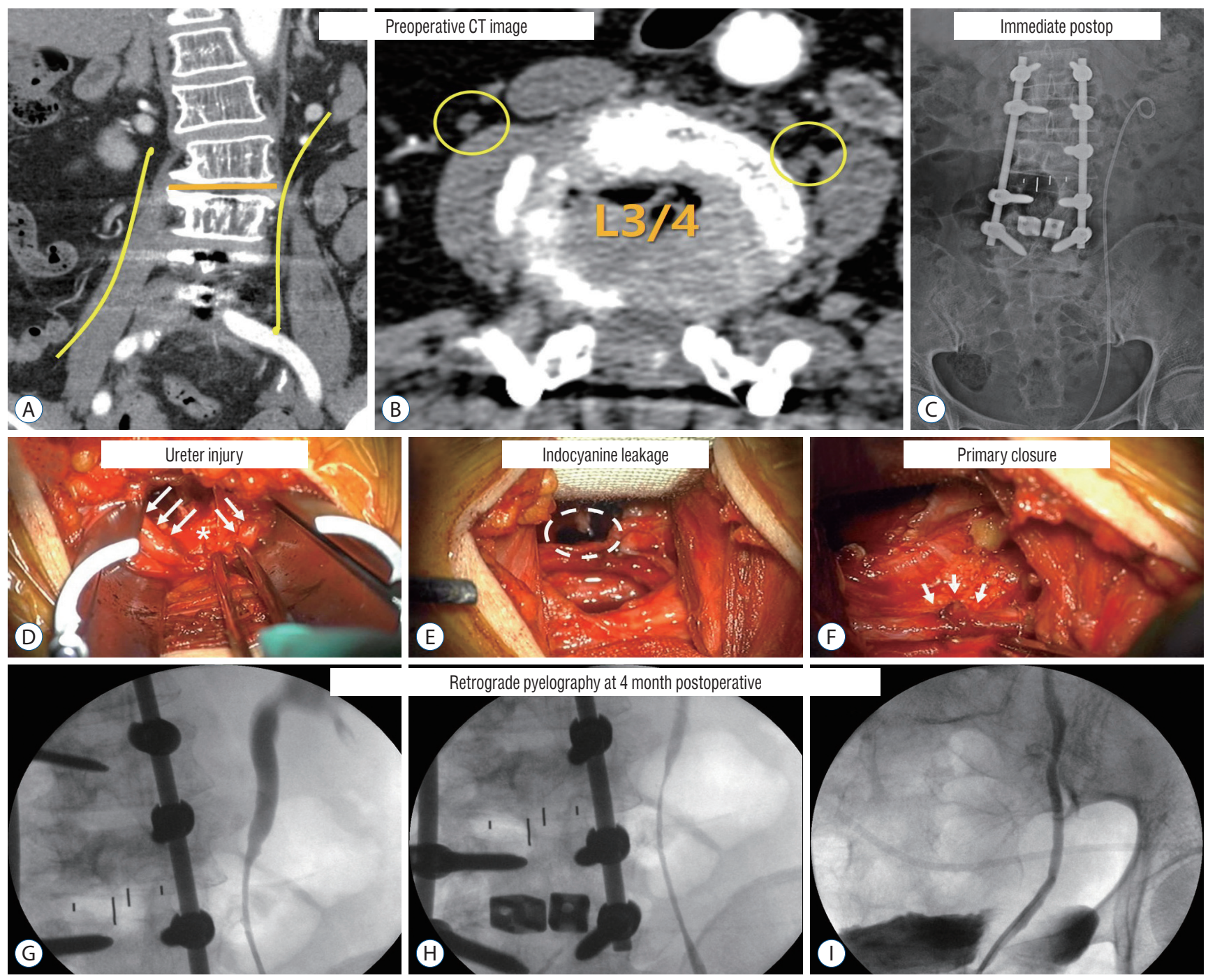

Fig. 6. Case with a complication of injury to the ureter. Preoperative CT image. A : The course of the ureter is indicated by the yellow line. B : The ureter at the $L 3 / 4$ area is marked with yellow circles. The right ureter is located above the psoas muscle, while the left ureter is located at the border of the vertebral body and the psoas muscle. Immediate postoperative image. C : L3-4 1 level OLIF with L1-L5 posterior fusion, left ureter with double J catheter inserted. Intraoperative images. D : Ureter (white arrows), injury site (asterisk). E : Indocyanine leakage was found at the site of injury of the ureter (dotted circle). F : A double J catheter was inserted, and primary closure was implemented (white arrows). Four months postoperative retrograde pyelography. G-I : Mild stenosis at the suture site; however, the contrast passage was good. CT : computed tomography, OLIF : oblique lateral interbody fusion.

was applied to the proximal and distal parts of the lumbar vein, respectively. After attaining complete hemostasis, the patient recovered well after surgery without any bleeding-related complications.

\section{DISCUSSION}

The occurrence of OLIF-related complications has been re- ported to range from $3.7 \%$ to $66.7 \%^{1-3,7-9,11,14,15,21,22,24,25,27,28,30,31)}$. In the meta-analysis studies of OLIF-related complications, psoas weakness (8.8\%) was the most common complication, followed by endplate fracture (5.2\%) and subsidence (5.1\%) ${ }^{19)}$. However, in our study, psoas paresis occurred more frequently (36.4\%) than in other studies; we believe that this was because the psoas muscle retraction time was long, and we were inexperienced in dissecting the anterior belly of the psoas muscle during the early stage of learning. In the binary logistic regres- 
sion analysis, both the 1-level (Fig. 2B) and 2-level (Fig. 2C) groups showed similar decreasing trends with case accumulation. In all patients (1-level+2-level), the probability decreased to less than 0.5 for approximately 50 cases (Fig. 2A). In addition, although we started OLIF later, we were carefully aware of the complications reported the literature and tried to record them accurately. In that respect, we assumed that psoas paresis occurred more frequently because many cases of grade 4 psoas paresis were included. Grade 4 psoas paresis (39/143, $27.3 \%$ ) had a considerably high incidence; accordingly, overall complications (56.4\%) were also higher than those in other studies. In addition, the incidence of remnant symptoms (30/143, 21\%) was higher than that reported in other studies. Except for these minor neurological complications, the overall complications were similar to those reported in other studies (18.9\%).

We found two studies on the learning curve of OLIF. Li et al. ${ }^{20)}$ compared the initial complications of direct lateral interbody fusion (DLIF) and OLIF. It was reported that the incidence of major complications was higher in OLIF than in DLIF (33.3\% vs. $10 \%, p=0.028)$. In particular, it emphasized the possibility of vessel injury in the early stages of learning. Another study compared the incidence of complications in the early stage (12 months) and late stage (14 months) of learn$\mathrm{ing}^{3)}$. As a result, the complication rate was $50 \%$ in the early stage; however, it decreased to $38 \%$ in the late stage. In addition, complications were reported steadily, even in the late stage. Our study also showed a learning curve of approach-related complications. Peritoneal lacerations and vessel injuries occurred frequently in the early stage; in particular, psoas paresis occurred at a high rate in the early stage (67.1\%). In the late stage, the incidence of psoas paresis significantly decreased (25.7\%). However, it is important to note that major complications such as cases requiring revision surgery and cases of injury to the ureter occurred intermittently even in the late stage.

Operation time and EBL are largely dependent on the surgical level and decompression. In that respect, the 3-level group with fewer cases was excluded, and the cases were divided into subgroups according to the use of decompression $(1 \mathrm{Lv}+\mathrm{D}$, $1 \mathrm{Lv}-\mathrm{D}, 2 \mathrm{Lv}+\mathrm{D}$, and $2 \mathrm{Lv}-\mathrm{D})$. The operative time in the decompression group showed large deviations due to various situations such as unilateral or bilateral decompression and cases requiring revision surgery. In the case of indirect decompres- sion, although statistical significance was not established, we found a decreasing trend in operation time. However, there was little difference in EBL between the early and late stages. This indicates that OLIF is a minimally invasive and easily accessible surgical method.

At our institution, patients are discharged when the CRP is normalized ${ }^{16)}$. Therefore, cases requiring revision due to infection are relatively rare. In the posterior approach, prolonged CRP normalization occurred in 26.1\% (24/92) of the cases and revision for abscess drainage was required in 3.2\% (3/92) of the cases ${ }^{13)}$. However, prolonged CRP normalization was found to be low at 4.9\% (7/143). We believe this result is due to the relatively small open operation field, minimal blood loss, and short operation time. In addition, in patients with elevated CRP, the revision rate was $12.5 \%(3 / 24)$ in the posterior approach, whereas it was $0 \%(0 / 7)$ in OLIF. These points indicate that OLIF shows better results in terms of infection compared with the conventional posterior approach.

Cage malposition has been reported in several experienc$\mathrm{es}^{18,20,30)}$. However, there is no detailed description of the risk factors and methods for managing this. Both the cases of cage malpositioning occurred when a cage of the usual size was used even though the insertion angle could not make a sufficient orthogonal. Huang et al. ${ }^{12)}$ recommended a relatively short and narrow cage when the orthogonality is not sufficient. In the review by Xu et al. ${ }^{29)}$, the authors recommend using a relatively small cage in OLIF than in DLIF to prevent contralateral root injury. In addition, when the cage is inserted in the C-arm without a true lateral image, significant cage rotation is observed on postoperative CT images ${ }^{6}$. We have to try to obtain the true lateral image of the C-arm, and if we cannot obtain the true lateral image due to severe deformity, we have to choose a relatively small cage. For revision surgery, the retroperitoneal approach has a limitation in that the possibility of endplate damage is high due to the orientation of the footprint of the cage. In addition, orthogonality could not be obtained due to high iliac crest in the primary surgery. It is challenging to change the angle during revision. In that respect, as per our experience, repositioning through a posterior approach is thought to be more advantageous.

Vertebral body fracture has been reported as a rare complication in the previous literature ${ }^{5,177}$. In our cases, vertebral coronal fracture occurred in patients with osteoporosis due to endplate breach and the use of a relatively large height cage. In 
particular, if the lower endplate of the upper vertebra has a concave shape, there is a high possibility of a breach during endplate preparation, and there is a risk of using a relatively large height cage to match the height of the center. In patients with osteoporosis with a concave endplate, more attention should be paid, and we recommend choosing a cage with a height that matches the lateral rim rather than the center of the disc.

Injury to the ureter occurred during the mid-stage. In most cases, since the ureter is located on or above the psoas, it is medially retracted during the retroperitoneal approach. However, in our case, the ureter was located between the psoas muscle and the vertebral body, and the ureter was not medially retracted. We mistook the ureter as a nerve fiber and placed it on the lateral side of the retractor, and damage occurred during the pulling out the trial after the discectomy. Fujibayashi et al. ${ }^{10)}$ reported that the ureter was located closest to the vertebral body at the proximal lumbar spine (L2-3). In addition, they recommend that confirming the course of the ureter on preoperative 2-phase abdominal CT can reduce this risk. If the ureter is located between the psoas muscle and the vertebral body, as in our case, the anterior border of the psoas muscle must be clearly dissected for accurately checking the ureter, following which the retractor should be installed.

This study has several limitations. First, factors related to clinical outcomes such as the Visual analogue scale (VAS) and the Oswestry disability index (ODI) were not described in this study. We measured these factors and found that most of the patients showed drastic improvement after surgery. However, our study consists of heterogeneous groups (multi-level, revision, various degenerative conditions, etc.). In that respect, the evaluation of the VAS and ODI changes was of little importance; hence, it was not described in this study. Second, this study did not include information about the fusion rate. Our study consisted of heterogeneous groups, and many patients had insufficient follow-up periods to evaluate the fusion rate. Nevertheless, this study describes in detail the changes in a series of OLIF as beginners and presents experiences of complications characteristically occurring in OLIF. We believe that it can provide a lot of information regarding OLIF to other beginners.

\section{CONCLUSION}

OLIF can be an easily accessible method without posing significant challenges during the early stage. According to the learning curve, the operation time (indirect decompression group) and psoas paresis decreased. Although some peritoneal laceration and vessel injury occurred in the early stage, continuous caution is required because injury to the ureter and cases requiring revision occur intermittently even in the late stage. It is important to select an appropriately sized cage along with clear dissection of the anterior border of the psoas muscle to prevent OLIF-specific complications, such as cage malposition, vertebral fracture, injury to the ureter, and vessel injury.

\section{CONFLICTS OF INTEREST}

Dong Wuk Son has been editorial board of JKNS since November 2017. He was not involved in the review process of this original article. No potential conflict of interest relevant to this article was reported.

\section{INFORMED CONSENT}

This type of study does not require informed consent.

\section{AUTHOR CONTRIBUTIONS}

\author{
Conceptualization : DWS, BKO, SHL \\ Data curation : DWS, SHL \\ Formal analysis : SHL, JSL \\ Funding acquisition : DWS \\ Methodology : BKO, SWL, GSS \\ Project administration : SKS, BKO, SHL \\ Visualization : SHL, BKO \\ Writing - original draft : BKO, SHL, DWS, SKS, SWL \\ Writing - review \& editing: DWS, GSS, SHL
}




\section{ORCID}

$\begin{array}{ll}\text { Bu Kwang Oh } & \text { https://orcid.org/0000-0002-6410-121X } \\ \text { Dong Wuk Son } & \text { https://orcid.org/0000-0002-9154-1923 } \\ \text { Su Hun Lee } & \text { https://orcid.org/0000-0001-8952-5556 } \\ \text { Jun Seok Lee } & \text { https://orcid.org/0000-0003-2488-6953 } \\ \text { Soon Ki Sung } & \text { https://orcid.org/0000-0001-7138-9621 } \\ \text { Sang Weon Lee } & \text { https://orcid.org/0000-0002-3199-7072 } \\ \text { Geun Sung Song } & \text { https://orcid.org/0000-0001-8029-9011 }\end{array}$

\section{- Acknowledgements}

This study was supported by Research institute for Convergence of biomedical science and technology (30-2020-008), Pusan National University Yangsan Hospital.

\section{References}

1. Abbasi $H$, Abbasi $A$ : Oblique lateral lumbar interbody fusion (OLLIF): technical notes and early results of a single surgeon comparative study. Cureus 7 : e351, 2015

2. Abbasi H, Miller L, Abbasi A, Orandi V, Khaghany K : Minimally invasive scoliosis surgery with oblique lateral lumbar interbody fusion: single surgeon feasibility study. Cureus 9 : e1389, 2017

3. Abe K, Orita S, Mannoji C, Motegi H, Aramomi M, Ishikawa T, et al. : Perioperative complications in 155 patients who underwent oblique lateral interbody fusion surgery: perspectives and indications from a retrospective, multicenter survey. Spine (Phila Pa 1976) 42 : 55-62, 2017

4. Boghani Z, Steele WI, Barber SM, Lee JJ, Sokunbi O, Blacklock JB, et al. Variability in the size of the retroperitoneal oblique corridor: a magnetic resonance imaging-based analysis. Surg Neurol Int 11 : 54, 2020

5. Brier-Jones JE, Palmer DK, Ǐnceoğlu S, Cheng WK : Vertebral body fractures after transpsoas interbody fusion procedures. Spine J 11 : 1068 1072, 2011

6. Chung NS, Lee HD, Jeon $\mathrm{CH}$ : Accuracy of the lateral cage placement under intraoperative C-arm fluoroscopy in oblique lateral interbody fusion. J Orthop Sci 23 : 918-922, 2018

7. DiGiorgio AM, Edwards CS, Virk MS, Mummaneni PV, Chou D : Stereotactic navigation for the prepsoas oblique lateral lumbar interbody fusion: technical note and case series. Neurosurg Focus 43 : E14, 2017

8. Fujibayashi $\mathrm{S}$, Hynes RA, Otsuki B, Kimura H, Takemoto M, Matsuda S: Effect of indirect neural decompression through oblique lateral interbody fusion for degenerative lumbar disease. Spine (Phila Pa 1976) 40 : E175-E182, 2015

9. Fujibayashi S, Kawakami N, Asazuma T, Ito M, Mizutani J, Nagashima $\mathrm{H}$, et al. : Complications associated with lateral interbody fusion: nation- wide survey of 2998 cases during the first 2 years of its use in Japan. Spine (Phila Pa 1976) 42 : 1478-1484, 2017

10. Fujibayashi S, Otsuki B, Kimura H, Tanida S, Masamoto K, Matsuda $S$ : Preoperative assessment of the ureter with dual-phase contrastenhanced computed tomography for lateral lumbar interbody fusion procedures. J Orthop Sci 22 : 420-424, 2017

11. Heo DH, Kim JS : Clinical and radiological outcomes of spinal endoscopic discectomy-assisted oblique lumbar interbody fusion: preliminary results. Neurosurg Focus 43 : E13, 2017

12. Huang $C, X u Z$, Li F, Chen Q : Does the access angle change the risk of approach-related complications in minimally invasive lateral lumbar interbody fusion? An MRI study. J Korean Neurosurg Soc 61 : 707715, 2018

13. Hwang JU, Son DW, Kang KT, Lee SH, Lee JS, Song GS, et al. : Importance of hemoglobin A1c levels for the detection of post-surgical infection following single-level lumbar posterior fusion in patients with diabetes. Korean J Neurotrauma 15 : 150-158, 2019

14. Jin C, Jaiswal MS, Jeun SS, Ryu KS, Hur JW, Kim JS : Outcomes of oblique lateral interbody fusion for degenerative lumbar disease in patients under or over 65 years of age. J Orthop Surg Res 13:38, 2018

15. Jin J, Ryu KS, Hur JW, Seong JH, Kim JS, Cho HJ : Comparative study of the difference of perioperative complication and radiologic results: MISDLIF (minimally invasive direct lateral lumbar interbody fusion) versus MIS-OLIF (minimally invasive oblique lateral lumbar interbody fusion). Clin Spine Surg 31 : 31-36, 2018

16. Kang KT, Son DW, Lee SH, Song GS, Sung SK, Lee SW : Variation of Creactive protein and white blood cell counts in spinal operation: primary fusion surgery versus revision fusion surgery. Korean J Spine 14 : 6670, 2017

17. Kim SB, Won YG, Lee JS, Ahn JS, Kang C, Lee GS : Vertebral body fracture after oblique lumbar interbody fusion in 2 patients: a case report. J Korean Soc Spine Surg 25 : 35-39, 2018

18. Kraiwattanapong C, Arnuntasupakul V, Kantawan R, Keorochana G, Lertudomphonwanit $\mathrm{T}$, Sirijaturaporn $\mathrm{P}$, et al. : Malposition of cage in minimally invasive oblique lumbar interbody fusion. Case Rep Orthop 2018 : 9142074, 2018

19. Li HM, Zhang RJ, Shen $\mathrm{CL}$ : Differences in radiographic and clinical outcomes of oblique lateral interbody fusion and lateral lumbar interbody fusion for degenerative lumbar disease: a meta-analysis. BMC Musculoskelet Disord 20 : 582, 2019

20. Li J, Wang X, Sun Y, Zhang F, Gao Y, Li Z, et al. : Safety analysis of two anterior lateral lumbar interbody fusions at the initial stage of learning curve. World Neurosurg 127 : e901-e909, 2019

21. Miscusi M, Ramieri A, Forcato $S$, Giuffrè $M$, Trungu $S$, Cimatti $M$, et al. : Comparison of pure lateral and oblique lateral inter-body fusion for treatment of lumbar degenerative disk disease: a multicentric cohort study. Eur Spine J 27(Suppl 2) : 222-228, 2018

22. Ohtori S, Orita S, Yamauchi K, Eguchi Y, Ochiai N, Kishida S, et al. : Miniopen anterior retroperitoneal lumbar interbody fusion: oblique lateral interbody fusion for lumbar spinal degeneration disease. Yonsei Med J 56 : 1051-1059, 2015 
23. Quillo-Olvera J, Lin GX, Jo HJ, Kim JS : Complications on minimally invasive oblique lumbar interbody fusion at L2-L5 levels: a review of the literature and surgical strategies. Ann Transl Med 6 : 101, 2018

24. Sato J, Ohtori S, Orita S, Yamauchi K, Eguchi Y, Ochiai N, et al. : Radiographic evaluation of indirect decompression of mini-open anterior retroperitoneal lumbar interbody fusion: oblique lateral interbody fusion for degenerated lumbar spondylolisthesis. Eur Spine J 26 : 671-678, 2017

25. Silvestre C, Mac-Thiong JM, Hilmi R, Roussouly P : Complications and morbidities of mini-open anterior retroperitoneal lumbar interbody fusion: oblique lumbar interbody fusion in 179 patients. Asian Spine J 6 : 8997, 2012

26. Suri P, Rainville J, Kalichman L, Katz JN : Does this older adult with lower extremity pain have the clinical syndrome of lumbar spinal stenosis? JAMA 304 : 2628-2636, 2010

27. Woods K, Fonseca A, Miller LE : Two-year outcomes from a single surgeon's learning curve experience of oblique lateral interbody fusion without intraoperative neuromonitoring. Cureus 9 : e1980, 2017

28. Woods KR, Billys JB, Hynes RA : Technical description of oblique lateral interbody fusion at L1-L5 (OLIF25) and at L5-S1 (OLIF51) and evaluation of complication and fusion rates. Spine J 17 : 545-553, 2017

29. Xu DS, Walker CT, Godzik J, Turner JD, Smith W, Uribe JS : Minimally invasive anterior, lateral, and oblique lumbar interbody fusion: a literature review. Ann Transl Med 6 : 104, 2018

30. Zeng ZY, Xu ZW, He DW, Zhao X, Ma WH, Ni WF, et al. : Complications and prevention strategies of oblique lateral interbody fusion technique. Orthop Surg $10:$ 98-106, 2018

31. Zhu G, Hao Y, Yu L, Cai Y, Yang X: Comparing stand-alone oblique lumbar interbody fusion with posterior lumbar interbody fusion for revision of rostral adjacent segment disease: a STROBE-compliant study. Medicine (Baltimore) 97 : e12680, 2018 